Mappemonde

Revue trimestrielle sur l'image géographique et les formes du territoire

$119 \mid 2017$

Varia

\title{
Le climat dans des images de paysage : éléments d'analyse
}

Alexis Metzger

OpenEdition

Journals

Édition électronique

URL : http://journals.openedition.org/mappemonde/2417

DOI : $10.4000 /$ mappemonde. 2417

ISSN : 1769-7298

Éditeur

UMR ESPACE

Référence électronique

Alexis Metzger, «Le climat dans des images de paysage : éléments d'analyse », Mappemonde [En ligne], 119 | 2017, mis en ligne le 01 janvier 2017, consulté le 14 septembre 2020. URL : http:// journals.openedition.org/mappemonde/2417

Ce document a été généré automatiquement le 14 septembre 2020

\section{(c) (i) (2)(2)}

La revue Mappemonde est mise à disposition selon les termes de la Licence Creative Commons Attribution - Pas d'Utilisation Commerciale - Partage dans les Mêmes Conditions 4.0 International. 


\title{
Le climat dans des images de paysage : éléments d'analyse
}

\author{
Alexis Metzger
}

\author{
Référence de la thèse \\ METZGER A. (2014). Le froid en Hollande au Siècle d'or. Essai de géoclimatologie culturelle. \\ Paris : Université Paris 1 Panthéon Sorbonne, thèse de doctorat de géographie, \\ $371 \mathrm{p}$.
}

1 Pour traquer le climat dans des images de paysage, le chercheur est en quête d'indices. En reprenant les analyses de notre thèse dirigée par Martine Tabeaud (Metzger, 2014), nous souhaitons dans cet article montrer comment le chercheur peut mener cette enquête, ce qu'il en coûte et ce qu'il y gagne. Ici, les images ne sont pas des illustrations aux résultats des recherches, elles sont la base de données analysée. Espérons que ce mini aide-mémoire sera par la suite amendé dans de nouveaux travaux !

\section{Constituer un corpus d'images}

2 Avoir des images en nombre suffisant est la condition sine qua non de toute analyse quelque peu significative. Si le chercheur veut faire des hypothèses climatologiques, il semble indispensable de prendre en considération des images montrant des paysages appartenant à la même échelle synoptique sur une période d'au moins trente ans. A fortiori, ces images doivent figurer des éléments météorologiques. Il n'est bien sûr pas inutile de se renseigner sur les auteurs de ces images, le courant artistique...

3 Notre corpus est composé de peintures de paysages hollandais du XVII ${ }^{\mathrm{e}}$ siècle, glanées au fil de la lecture de catalogues d'expositions et de visites (un exemple en figure 1). Les Pays-Bas sont un pays où les types de temps sont assez homogènes, malgré des gradients entre l'ouest et l'est et le sud et le nord. À cette époque, la peinture de paysage connait un essor formidable aux Pays-Bas, particulièrement dans la province de Hollande. Nous avons choisi de nous concentrer sur une saison, l'hiver. 
Figure 1. Adam van Breen, Paysage d'hiver avec un canal gelé, 1611, Rijksmuseum, Amsterdam

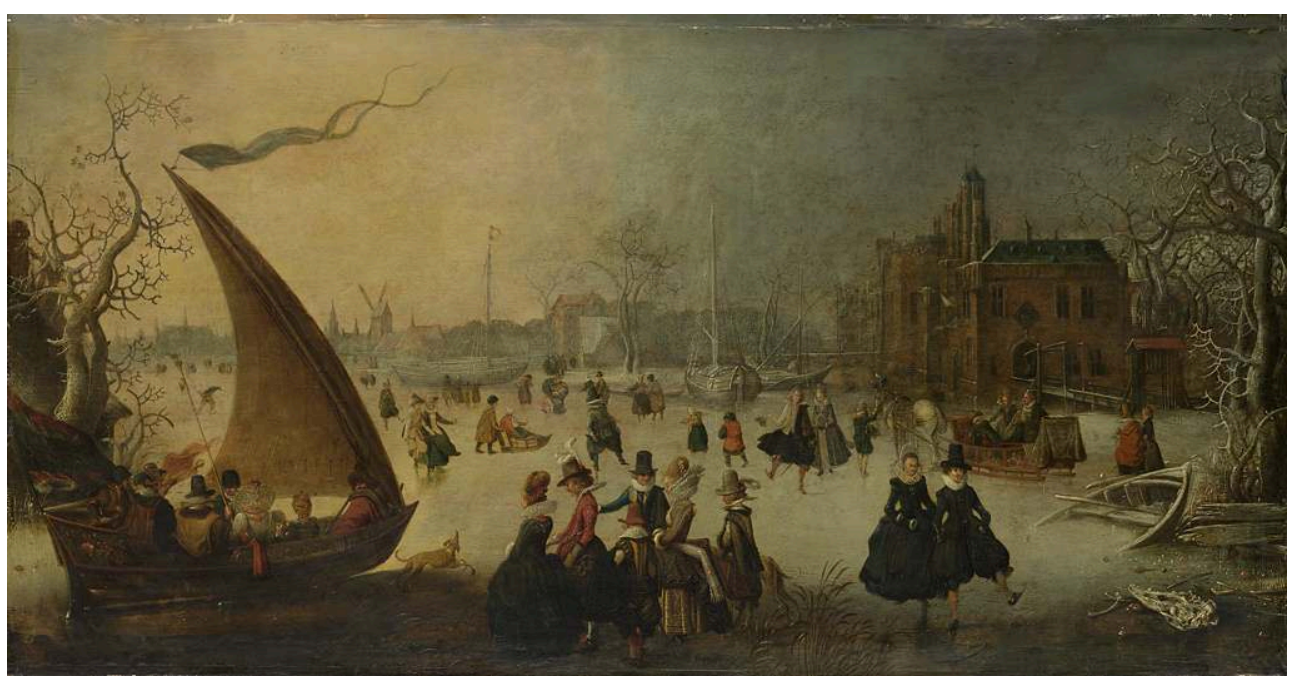

\section{Rassembler des données climatologiques}

4 Appartenant à la même échelle synoptique que les paysages précédemment mentionnés, ces données doivent être les plus riches possibles. Cette récolte peut être fastidieuse si le corpus d'images date d'avant la systématisation des observations dans les stations météo normées.

5 Notre corpus datant du XVII ${ }^{\mathrm{e}}$ siècle, il n'existait pas de relevés systématisés. Il a donc été nécessaire de recourir aux travaux d'historiens du climat (par exemple, Buisman, 2000) et d'analyser très précisément certains carnets météorologiques tenus par des amateurs passionnés (Metzger et Tabeaud, 2012).

\section{Faire l'inventaire des marqueurs paysagers}

Faute de données chiffrées comparables entre elles (et par rapport à un étalon), le travail consiste à inventorier l'ensemble des marqueurs paysagers se référant au temps qu'il fait dans les images de paysages (voir aussi Tabeaud et Lysianuk, 2009). Le chercheur s'intéressera par exemple aux marqueurs de saisonnalité, aux météores, aux attitudes humaines pouvant être liées aux types de temps... Cette étape ne permet pas la reconstitution du climat mais elle permet de définir le type de temps des images en assemblant des éléments visuels comme les pièces d'un puzzle.

7 Un grand tableau renseignant notamment les éléments composant le type de temps a été progressivement rempli. Nous avons par exemple indiqué les types de nuages identifiables... il peut être intéressant d'estimer l'heure de la journée et la position du soleil. Ces indications donnent les points cardinaux de l'image et permettent alors de connaitre l'orientation du vent et donc envisager des flux synoptiques s'ils sont identiques à l'orientation du vent observée au sol. Comme dit Roland Barthes en parlant des scènes de patinage peintes par Avercamp, le grand spécialiste des scènes d'hiver dans la Hollande du Siècle d'or, «il faut déchiffrer cela comme on lit un 
rébus »! Nous pouvons par exemple inventorier sur la figure 1 les observations suivantes (tableau 1, aux entrées adaptées à notre corpus hivernal) :

Tableau 1. Inventaire des marqueurs paysagers de la figure 1

\begin{tabular}{|c|c|c|}
\hline Toile & Peintre & Adam van Breen \\
\hline & Nom de la peinture & Paysage d'hiver avec un canal gelé \\
\hline & Collection/Musée & Rijksmuseum, Amsterdam \\
\hline \multirow[t]{10}{*}{ Type de temps } & Ensoleillé? & Non \\
\hline & Nuages? & Oui \\
\hline & Types de nuages? & Stratus \\
\hline & Couleur du ciel & Ocre jaune \\
\hline & Vent? & Oui, fort apparemment \\
\hline & Pluie? & Non \\
\hline & Manteau neigeux? & À peine \\
\hline & Épaisseur? & Fine pellicule de neige \\
\hline & Glace? & Oui \\
\hline & Épaisseur? & Importante \\
\hline \multirow[t]{4}{*}{ Espace } & Habitat & Ville en arrière-plan \\
\hline & Moulin & Oui, 1 \\
\hline & Eau & Oui, cours d'eau \\
\hline & Relief? & Non \\
\hline \multirow[t]{8}{*}{ Activités } & Animaux? & 2 chiens, 1 cheval, $(+1$ cadavre de cheval au premier plan?) \\
\hline & Nombre d'habitants & Nombreux \\
\hline & Catégorie sociale & Melting-pot mais aisés au premier plan \\
\hline & Patinage? & Oui \\
\hline & Jeux? & Non \\
\hline & Déplacements? & Traineaux de 2 tailles différentes, char à voile sur la glace \\
\hline & Feu? & Non \\
\hline & Pêcher? & Non \\
\hline
\end{tabular}




\begin{tabular}{|l|l|l|}
\hline & Ramasser du bois ? & Non \\
\hline & Autre? & \\
\hline
\end{tabular}

\section{Quantifier les observations qualitatives}

8 À partir du moment où le corpus d'images est suffisant, il est possible de quantifier l'indexation faite lors de l'étape précédente en faisant des pourcentages (combien d'images montrent de la pluie, du vent, etc.). L'idée est de parvenir à synthétiser une représentation moyenne des types de temps dans le corpus choisi pour dégager le climat de la période du corpus.

9 Nous avons alors abouti à des pourcentages de peintures montrant ou pas un ciel dégagé, un manteau neigeux, des personnes courbées marchant contre le vent... Notons sur notre peinture (figure 1) une incohérence : apparemment le vent vient de la gauche du tableau, gonflant la toile de la barque sur patins mais juste derrière, un homme courbé semble marcher contre le vent... qui viendrait de droite. Le peintre a dû procéder par juxtaposition de petites scènes indépendantes les unes des autres.

\section{Faire une comparaison entre le climat représenté et le climat relevé}

10 Se pose alors une question clef : dans quelle mesure les images montrent bel et bien le climat d'une région donnée? Pour y répondre, il est nécessaire de se pencher vers les surreprésentations ou sous-représentations de certains paramètres.

11 Il est apparu que les peintures hollandaises hivernales surreprésentent la glace (tous les cours d'eau et plans d'eau sont gelés dans les tableaux!) mais ne montrent pas d'autres météores (la pluie, la chute de neige...). Le chercheur peut comparer ces pourcentages aux seules observations météorologiques faites quotidiennement pendant notre période sur plus de 10 ans, celles du pasteur David Fabricius. Le tableau cidessous (tableau 2) montre, par exemple, les principaux types de temps déduits du carnet. Or, dans les peintures « hivernales » les types A, C, E et F sont très rares !

Tableau 2. Fréquence des types de temps entre 1594 et 1612 d'après les observations de David Fabricius, pasteur résidant en Frise-Orientale

\begin{tabular}{|l|l|}
\hline A gel avec précipitations & $5,5 \%$ \\
\hline B gel sans précipitations & $16,8 \%$ \\
\hline C alternance gel-dégel avec précipitations & $12 \%$ \\
\hline D alternance gel-dégel sans précipitations & $14,2 \%$ \\
\hline E pas de gel avec précipitations & $26,1 \%$ \\
\hline F pas de gel sans précipitations & $25,4 \%$ \\
\hline
\end{tabular}




\section{Interroger les choix climatiques des images} posent. Quels sont les auteurs et les destinataires des images? Dans quel contexte s'inscrivent ces images? Ces questions simples permettent d'éclairer les choix des éléments climatiques faits par les auteurs des images.

artistes du Siècle d'or peignaient majoritairement pour les nouveaux bourgeois hollandais. Alors que la province de Hollande appartient aux Provinces-Unies quasiindépendantes de l'Espagne depuis la Trêve de 1609, il pouvait s'agir pour les peintres de représenter une nouvelle nation libre et heureuse de se montrer à l'extérieur. D'où l'absence de précipitations. La présence de la glace renforce le melting-pot: riches et pauvres patinent ensemble, les pauvres n'ont pas à payer les déplacements estivaux en barges. Enfin, patiner sur la glace, c'est aussi dominer l'eau (figée) qui peut être destructrice lors de grandes inondations. Les conclusions de cette thèse trouvent donc des échos aussi bien en climatologie historique ou géographie historique qu'en histoire de l'art.

\section{Pistes à développer}

- Si le corpus d'images est étendu dans le temps (au moins deux périodes normales de trente ans), on peut tenter de déceler des variations climatiques entre les images de deux différentes périodes climatiques. Ces variations dans les représentations correspondentelles avec les variabilités relevées? Pourquoi?

- On peut choisir deux corpus appartenant à une même échelle synoptique mais à deux autres découpages (politique, administratif, culturel...) et voir en quoi les représentations du climat diffèrent ou non dans les images.

- Peut-on choisir un corpus d'images montrant difFérentes saisons? Oui, sans doute, mais il semble indispensable de construire toutes les étapes indépendamment pour chaque saison. On pourra alors in fine se demander si les sur ou sous-représentations sont les mêmes pour chaque saison ou non.

- Cette méthode doit pouvoir être applicable à un corpus de textes. Parcourir ces étapes en ayant en poche des textes littéraires, par exemple, parait tout à fait envisageable même si l'indexation des marqueurs peut prendre en ce cas beaucoup plus de temps (qui passe)!

\section{BIBLIOGRAPHIE}

BUISMAN J. (2000). Duizend jaar weer, Wind en Water in de Lage Landen, volume IV : 1575-1675. La Haye : Wijnen/KNMI, 767 p. ISBN 9789051941432

METZGER A., TABEAUD M. (2013). « Les météophiles hollandais de la neige au XVII ${ }^{\mathrm{e}}$ siècle ». In DUCOS J. (dir.), Météores et climats d'hier, Paris : Hermann, p. 99-123. ISBN 9782705684600

Mappemonde, $119 \mid 2017$ 
TABEAUD M., LYSIANUK B. (2009). « L'Europe en images... à la manière des offices de tourisme ». Physio-Géo, vol. 3.

INDEX

Mots-clés : prix de thèse 2015

\section{AUTEUR}

ALEXIS METZGER

Université de Limoges 